\title{
The Potential of Science and Technology Innovation in the Future Development of Media
}

\author{
Chen Qiyi \\ Shanghai Rongyue Private Entry-Exit Services Co., Ltd, 200000, China
}

Keywords: science and technology innovation, media, future development, new opportunities

\begin{abstract}
Science and technology play a decisive role in the iterative innovation of media industry. And the innovation of science and technology will bring many new opportunities to the media industry. For example, enriching the media matrix of mobile terminal will become the trend, also the news report based on virtual reality may become the mainstream, and the public domain of media network will be constructed.
\end{abstract}

Science and technology should be the first productive force and they play a decisive role in innovation and development of media industry. It will also bring new opportunities to the media.

\section{Technology-driven mechanism of transmission}

Throughout the history of media development and practice, science and technology would be the most fundamental force, which is mainly reflected by the following three mechanisms. Firstly, science and technology would greatly reduce the difficulty of the audience and users to contact and participate in the media. Secondly, science and technology drive economic development and social progress, so as to improve people's knowledge and culture level. Thirdly, science and technology make people have more leisure time and can enjoy more media products. And the invention of characters, Gutenberg and movable type printing, radio and television technology have brought fundamental changes to the media industry. In particular, the Internet has brought essential changes to the media.

\subsection{Internet technology has achieved equal rights in technology}

On the one hand, it breaks the situation that information is controlled by elites, which gives ordinary people the right to spread information to a great extent. On the other hand, due to the emergence of social media, information increases rapidly. Internet technology has broken the communication restrictions controlled by elites, making it possible for us to communicate timely and interactively.

\subsection{The Internet has greatly enriched the content and scope of the media}

First of all, the existing media can be transformed into the Internet form, such as Internet news, Internet books, Internet video, Internet audio, and Internet animation. Secondly, it greatly expands the crowd and scope of media influence, bringing hundreds of users into the scope of media services. Thirdly, Internet media has become the main channel of public cultural services and other cultural services. In particular, the Internet platform has built a bridge of communication between different subcultures. The subcultures which were relatively low in civilization but could not be paid attention to by the outside world due to their relative closeness were put under the attention of all circles, thus forcing the subcultures with lower civilization to evolve continuously.

\subsection{The Internet media industry promotes the development and prosperity of China's media industry}

The Internet media industry has not only become the core component of cultural industry, but also become the core driving force of the development of cultural industry. In addition, on the map of the world's enterprises of Internet media, Tencent, Alibaba, Baidu, iQiyi and so on are playing an 
increasingly important role. In particular, the enterprises of Internet media integrating technology and cultural creativity are forcing and promoting the transformation and upgrading of China's traditional industries, so as to better carry out the sustainable development of the whole economy.

\section{New opportunities for media in the era of scientific and technological innovation}

When it comes to China's scientific and technological innovation, one has to mention the following two words, what are, artificial intelligence and 5G. As we know, 5G massive MIMO and dense small cells can support more than 1 million devices per square kilometer. It is often said that 4G has changed the way people communicate, while 5G has deepened the relationship between media and society and shaped autonomous organizations. With $5 G$ as the representative, this paper expounds the potential of technological innovation in the future development of media.

\subsection{Enriching the media matrix of mobile terminal}

The statistical report on the development of Internet shows the development of Internet and its users in China. The existing stock of users is also the consumer base for the extended development of media. According to the 43rd statistical report on the development of China's Internet in 2019, as of June 2019, the number of Internet users in China has reached 854 million, with 847 million mobile Internet users, an increase of 3.04\% and 3.52\% over the end of 2018. Almost all users will use mobile phones to access the Internet. On the one hand, the development of network information technology makes new media occupy the leading position in the media industry. On the other hand, the commercial support of 5G will further enrich the users' real-time experience of mobile terminal and make mobile terminal become the mainstream of media. Therefore, in the 5G eras, the reconstruction of relationship will become a new starting point, and enriching the product matrix of mobile terminal with $5 \mathrm{G}$ technology will become a new starting point to solve the problem of loss of traditional users. At the same time, it is also necessary to improve consumer experience, enhance the stickiness between users and products, and complete the transformation and upgrading from "print media" to "immersive media".

\subsection{Virtual reality based news reporting may become mainstream}

The breakthrough of communication technology will promote the development of news presentation from pictures and texts to video and virtual reality. The video news broadcast based on impressiveness will become the mainstream of news reporting. This change will make readers get the most needed information anytime, anywhere, intuitively and accurately. In the future, facing the $5 G$ eras, traditional media should keep up with the development direction of technology, update and upgrade the production mode of news content, deepen the process of media integration, and then explore and realize new business ecology and profit model. Taking VR news broadcast as an example, customized VR makes users feel like they are on the news scene, and can feel the news environment and content in multiple dimensions. Users have also become participants of news events, "on-the-spot witnesses", and even the central figures promoting the development of news events, rather than just consumers and "outsiders" of news events.

\subsection{Using big data to build the public domain of media network}

After the deep integration of the Internet and the media industry in the $4 \mathrm{G}$ era, the entry barriers of the media industry have been broken. Massive user resources, diversified production modes, complex communication structures and diverse terminals announce that the media ecology has ushered in the "mass media era". On the one hand, the diversification of media causes the generalization of media attributes, and the lack of strict examination and control of information sources by new media platforms and communicators, resulting in "a lot of noise". On the other hand, with the support of big data and artificial intelligence technology, new media has the ability of user portrait and data tracking. In the 5G eras, data tracking and matching technology will become more accurate and mature, and further highlight the opposition of groups. In view of this, traditional media should give full play to the credibility of mainstream media, actively report in the public 
domain, and provide users with a broad space for information collision and in-depth communication and exploration. In this platform, the fairness of news reports, the right of the public to know, the right to speak, and the fairness of public opinion will be realized to the maximum extent. At the same time, the construction of the media platform should strengthen the classification and review of information push.

\subsection{Grasping the data explosion and improving the ability of public opinion monitoring}

In the $5 \mathrm{G}$ eras, the exponential growth of information, pictures, and video data is transmitted more quickly and conveniently by means of intelligent perception and information collection. At the same time, with the spread of real-time data, public opinion also generated, fermented, exploded and declined with the same exponential growth rate. The Internet of all things media would bring about massive and rapid online public opinion monitoring, which brings difficulties and opportunities for more accurate and real-time public opinion monitoring. Firstly, a holographic database covering all kinds of intelligent terminals should be established. Secondly, the real-time monitoring ability of monitoring system of public opinion should be strengthened, and the whole public opinion monitoring process should be "Digitized" after the event, so as to adapt to 5G. Finally, building an independent online red flow team to actively attack and accurately respond to public opinion.

With the advent of the $5 \mathrm{G}$ eras, the media industry bid farewell to the economy brought by strong capital, technical support and keen market insight, and ushered in the second half of media convergence and competition. The coming of the 5G eras will largely make up for the weakening of influence and the loss of users and audiences caused by the "channel failure" of traditional media in the Internet era.

To sum up, the traditional media should do the following four "persistence" in order to truly seize the development opportunity of traditional media given by the era of scientific and technological innovation. Firstly, we should adhere to build a solid technical foundation and focus on building a mobile media position. Secondly, we should persist in the principle of content as the core, and strive to build public territory of media network, also undertake the responsibility of mainstream media, as well as enhancing the credibility of media. Thirdly, we should uphold the priority of user experience, enrich the social, intelligent and visual product matrix, and increase user stickiness. Last but not least, we should manage to stick to the people-oriented thinking logic, enabling the transformation of media science and technology.

\section{References}

[1] Tong Wen. What capabilities does $5 G$ have? [EB/OL]. https://baijiahao.baidu.com/s?id=1623233462134077356\&wfr=spider\&for=pc,2019-01-21/2019-04 $-02$.

[2] Guo Quanzhong. Science and technology drive the development of culture. [J]. Young reporter, September 2019. 